\title{
THE EFFECT OF LIGHT BENDING AND REDSHIFT ON PULSAR BEAMING: THE CASE OF SHORTER ROTATION PERIODS
}

\author{
R. C. KAPOOR \\ Indian Institute of Astrophysics
}

\begin{abstract}
An estimate of the effect of light bending and redshift on pulsar beam characteristics has been made using a weak Kerr metric for the case of a $1.4 \mathrm{M}_{\odot}$ neutron star with a radius in the range $6-10 \mathrm{~km}$ and rotation periods of $1.56 \mathrm{~ms}$ and $33 \mathrm{~ms}$, respectively. Assuming that the pulsar emission has the form of a narrow conical beam directed away from the surface and is located within two stellar radii, the beam is found to be widened by a factor of $\leq 2$ and to suffer a reduction in the intensity (flattening of the profile) by an order of magnitude or less. The effect is largest for the most rapidly rotating the neutron stars. For an emission region located beyond $20 \mathrm{~km}$, the flattening is generally insignificant. The pulse profile is slightly asymmetrical due to dragging of the inertial frames. For millisecond periods, aberration tends to reverse the flattening effect of space-time curvature by narrowing the pulse and can completely overcome it for emission from a location beyond $\simeq 30 \mathrm{~km}$. Although the pulse must slightly brighten up, a large redshift factor overcomes this effect to keep the pulse flattened for all neutron star radii considered here.
\end{abstract}

\section{Photon propagation near neutron star}

Our purpose here is to report results of a calculation to estimate the contribution of light bending and redshift to the pulsar beaming characteristics where the pulsar mass is $1.4 \mathrm{M}_{\odot}$, but the radius lies in the range $6-10 \mathrm{~km}$. The case for smaller neutronstar radii has been made by Mészáros and Riffert (1987) in the course interpreting observational evidence on X-ray pulsars and QPOs. With a slight modification, we use the basic formalism developed in Kapoor and Datta (1985) which refers to the conventional model of pulsar emission proposed by Radhakrishnan and Cooke (1969). The conical pulsar beam, regarded monochromatic here, is widened due to space-time curvature in longitude (and latitude). Here a photon emitted from $r=r_{\text {em }}$ at an angle $\delta$ with respect to the radius vector arrives at a distant observer at an angle $\delta_{\text {new }}$-widening the beam in that $\left|\delta_{\text {new }}\right|>|\delta|$. The angle $\delta$ is measured in the direction opposite to that of the rotation of the neutron $\operatorname{star}(\delta=-\pi / 2$ represents a tangential forward photon, $\delta=0$ is radially outward, etc.), whereas the angle $\delta_{\text {new }}$ is the same as the net bending of a photon $\varphi_{0}$ according to an observer at infinity. This quantity can be evaluated once one explicitly writes down the geodesic equations corresponding to the weak Kerr geometry

$$
\varphi_{0}(\delta=0)-\varphi_{0}(\delta)=\delta_{\text {new }},
$$

where

$$
-\varphi_{0}=\int \frac{d \varphi / d \Gamma}{d r / d \Gamma} d r
$$

a $\Gamma$ being an affine parameter. The beam is symmetrically widened only in the Schwarzschild case [where $\varphi_{0}(\delta=0)=0$ ]; in the rotational case, photons emitted at an angle $\delta$ are slightly swept away in the direction of rotation of the star as a consequence of the frame drag effect. As a measure of the widening of the beam, we introduce a quantity 'divergence index'

$$
\Delta=\frac{d \delta_{\text {new }}}{d \delta}=-\left.\frac{d \varphi_{0}}{d \delta}\right|_{\text {rem }}
$$

The $\Delta$ in eq.(3) differs from that defined in Kapoor and Datta (1985) where the angle $\delta$ was redundantly added to $\varphi_{0}$ to give $\delta_{\text {new }}$. In the rotational case we find that $\Delta(+\delta) \neq \Delta(-\delta)$. There is a reduction in the intensity of the pulse due to the beam widening which is measured by a deamplification factor

$$
\epsilon=\frac{\sin \delta}{\sin \delta_{\text {new }}} \frac{d \delta}{d \delta_{\text {new }}}=\frac{1}{\Delta} \frac{\sin \delta}{\sin \delta_{\text {new }}}
$$

It will be $<1$ when the beam is widened. Since there is an inherent reduction in intensity due to redshift also, the net reduced intensity as observed at infinity is

$$
I_{r}^{ \pm}=I_{e} \frac{\epsilon}{[1+z( \pm \delta)]^{3}},
$$

where $I_{e}$ is the intensity at the emission frequency in the rest frame of the emitter. Due to slight Doppler boosting and diminution respectively, $I_{\mathrm{r}}^{-}>I_{\mathrm{r}}^{+}$. 
Table 1

\begin{tabular}{|c|c|c|c|c|c|c|c|c|c|}
\hline$R(\mathrm{~km})$ & V./e & $\varphi_{0}\left(+5^{6}\right)$ & $Z^{+}$ & $\Delta^{+}$ & $I_{r}^{+} / I_{\mathrm{a}}$ & $\varphi_{0}\left(-5^{0}\right)$ & $Z^{-}$ & $\Delta^{-}$ & $I_{\mathrm{r}}^{-} / I_{\mathrm{e}}$ \\
\hline \multicolumn{10}{|c|}{$P=1.56 \mathrm{~ms}, \theta_{0}=\pi / 2($ weak Kerr $) \quad r_{e=-}=R$} \\
\hline 6 & 105 & -8.785 & 6.343 & 1.747 & .051 & 8.909 & 5.896 & 1.797 & 053 \\
\hline 7 & 112 & -7.643 & 4.231 & 1.519 & .102 & 7.757 & 3.929 & 1.564 & .05 \\
\hline 8 & .123 & -7.018 & 3.343 & 1.394 & .153 & 7.129 & 3.093 & 1.438 & .156 \\
\hline 9 & .134 & -6.617 & 2.870 & 1.313 & 201 & 6.728 & 2.644 & 1.358 & 207 \\
\hline 10 & .147 & -6.334 & 2.586 & 1.256 & .243 & 6.447 & 2.371 & 1.301 & 252 \\
\hline \multicolumn{10}{|c|}{$P=1.56 \mathrm{~ms}, \theta_{0}=\pi / 2($ Schwarzschild $)$} \\
\hline 6 & & -9.010 & 6.176 & 1.805 & .050 & & 5.724 & 1.805 & .054 \\
\hline 7 & & -7.837 & 4.097 & 1.569 & .099 & & 3.767 & 1.569 & .108 \\
\hline 8 & & -7.206 & 3.213 & 1.442 & .150 & & 2.962 & 1.442 & .163 \\
\hline 0 & & -6.808 & 2.735 & 1.362 & 197 & & 2.509 & 1.362 & .215 \\
\hline 10 & & -6.533 & 2.441 & 1.307 & .240 & & 2.227 & 1.307 & .263 \\
\hline \multicolumn{10}{|c|}{$P=33 \mathrm{~ms}, \theta_{0}=\pi / 2($ weak Kerr $)$} \\
\hline 6 & .005 & -8.974 & 5.772 & 1.797 & .054 & 8.979 & 5.752 & 1.799 & .054 \\
\hline 7 & .005 & -7.815 & 3.823 & 1.563 & 107 & 7.820 & 3.809 & 1.566 & 107 \\
\hline 8 & .006 & -7.190 & 2.982 & 1.439 & .162 & 7.195 & 2.970 & 1.441 & .163 \\
\hline 0 & .006 & -6.796 & 2.520 & 1.359 & .215 & 6.801 & 2.510 & 1.361 & .215 \\
\hline 10 & .007 & -6.523 & 2.231 & 1.305 & .264 & 6.528 & 2.221 & 1.307 & .264 \\
\hline
\end{tabular}

Table 2

\begin{tabular}{|c|c|c|c|c|c|c|c|c|c|}
\hline$R(\mathbf{k m})$ & $V . / c$ & $\varphi_{0}\left(+5^{\circ}\right)$ & $Z^{+}$ & $\Delta^{+}$ & $I_{\mathrm{r}}^{+} / I_{\mathrm{e}}$ & $\varphi_{0}\left(-5^{\circ}\right)$ & $Z^{-}$ & $\Delta^{-}$ & $I_{\mathrm{r}}^{-} / I_{\mathrm{e}}$ \\
\hline \multicolumn{10}{|c|}{$P=1.56 \mathrm{~ms}, \theta_{0}=\pi / 2($ weak Kerr $) \quad r_{e m}=2 R$} \\
\hline 6 & 193 & -6.000 & 2.339 & 1.191 & .299 & 6.098 & 2.124 & 1.230 & .314 \\
\hline 7 & 218 & -5.746 & 2.208 & 1.139 & .346 & 5.854 & 1.985 & 1.182 & .364 \\
\hline 8 & .244 & -5.557 & 2.166 & 1.100 & 378 & 5.675 & 1.929 & 1.147 & 398 \\
\hline 9 & .270 & -5.405 & 2.180 & 1.009 & 397 & 5.532 & 1.925 & 1.120 & 419 \\
\hline 10 & .296 & -5.275 & 2.236 & 1.042 & .407 & 5.412 & 1.959 & 1.097 & 430 \\
\hline \multicolumn{10}{|c|}{$P=1.56 \mathrm{~ms}, \theta_{0}=\pi / 2($ Schwarzschild $)$} \\
\hline 6 & & -6.177 & 2.108 & 1.236 & .311 & & 1.899 & 1.236 & .345 \\
\hline 7 & & -5.955 & 1.936 & 1.194 & .364 & & 1.721 & 1.194 & .409 \\
\hline 8 & & -5.803 & 1.840 & 1.161 & .404 & & 1.614 & 1.161 & .460 \\
\hline 9 & & -5.692 & 1.789 & 1.139 & .431 & & 1.548 & 1.139 & .498 \\
\hline 10 & & -5.608 & 1.766 & 1.122 & .450 & & 1.508 & 1.122 & .527 \\
\hline \multicolumn{10}{|c|}{$P=1.56 \mathrm{~ms}, \theta_{0}=\pi / 2($ weak Kerr $)$} \\
\hline 6 & .010 & -6.169 & 1.889 & 1.234 & .348 & 6.174 & 1.880 & 1.236 & .349 \\
\hline 7 & .010 & -5.948 & 1.696 & 1.189 & .417 & 5.953 & 1.686 & 1.191 & .418 \\
\hline 8 & .011 & -5.797 & 1.571 & 1.159 & .474 & 5.802 & 1.562 & 1.161 & .475 \\
\hline 9 & .013 & -5.686 & 1.485 & 1.137 & .521 & 5.693 & 1.475 & 1.139 & .523 \\
\hline 10 & .014 & -5.602 & 1.422 & 1.120 & .561 & 5.609 & 1.411 & 1.123 & .563 \\
\hline
\end{tabular}

\section{Results and conclusions}

To estimate the effect of spacetime curvature and rotation on the pulse profile and the beaming, we choose $M=1.4 M_{a}\left(R_{0}=4.1 \mathrm{~km}\right), R=6-10 \mathrm{~km}$, and $P$ values of both $33 \mathrm{~ms}$ and $1.56 \mathrm{~ms}$ were considered. The results are given in tables 1-4 which correspond to various locations of the emitter and slightly differ from those in Kapoor and Datta (1985) as a result of the modified form of $J$ in eq.(3). The main feature we notice is that the effect of space-time curvature is the strongest for he shortest period neutron star, where the beam is widened by a factor of $\sim 2$ and the intensity reduced by an order of magnitude for $r_{\mathrm{em}}=R$. In general, the larger the neutron star, the less is the widening effect on the beam caused by space-time curvature. The widening effect on the beam dwindles rapidly as $r_{\mathrm{em}}$ increases beyond $20 \mathrm{~km}$. The attendant reduction in intensity is likewise minimal.

The situation for $P=1.56 \mathrm{~ms}$, however, is distinguished for all the radii considered. Here, due to the presence of rotational terms in the formalism, the aberration effect is nontrivial. more so where $r_{\mathrm{em}}$ is large. The main function of aberration is 
Table 3

\begin{tabular}{|c|c|c|c|c|c|c|c|c|c|}
\hline$\overline{R(k m)}$ & $\overline{V . / c}$ & $\varphi_{0}\left(+5^{\circ}\right)$ & $Z^{+}$ & $\Delta^{7}$ & $I_{\mathrm{r}}^{+} / I_{\mathrm{g}}$ & $\varphi_{0}\left(-5^{\circ}\right)$ & $Z^{-}$ & $\Delta^{-}$ & $I_{\mathrm{r}}^{-} / I_{0}$ \\
\hline \multicolumn{10}{|c|}{$P=1.56 \mathrm{~ms}, \theta_{0}=\pi / 2($ weak Kerr $) \quad r_{00}=3 R$} \\
\hline 6 & .273 & -5.416 & 2.193 & 1.071 & .393 & 5.541 & 1.937 & 1.121 & .416 \\
\hline 7 & .312 & -5.227 & 2.292 & 1.032 & .404 & 5.367 & 2.002 & 1.088 & 428 \\
\hline 8 & .352 & -5.063 & 2.470 & 0.998 & .401 & 5.217 & 2.138 & 1.059 & 423 \\
\hline 9 & .392 & -4.911 & 2.726 & 0.966 & .387 & 5.077 & 2.343 & 1.033 & 497 \\
\hline 10 & .432 & -4.762 & 3.070 & 0.935 & .366 & 4.911 & 2.628 & 1.007 & .382 \\
\hline \multicolumn{10}{|c|}{$P=1.56 \mathrm{~ms}, \theta_{1}=\pi / 2($ Schwarzschild $)$} \\
\hline 6 & & -5.693 & 1.789 & 1.139 & .431 & & 1.548 & 1.139 & 498 \\
\hline 7 & & -5.573 & 1.763 & 1.115 & .457 & & 1.495 & 1.115 & .538 \\
\hline 8 & & -5.488 & 1.780 & 1.098 & .466 & & 1.479 & 1.098 & .561 \\
\hline 9 & & -5.426 & 1.831 & 1.085 & .464 & & 1.489 & 1.085 & .571 \\
\hline 10 & & -5.375 & 1.912 & 1.075 & .453 & & 1.523 & 1.075 & .568 \\
\hline \multicolumn{10}{|c|}{$P=1.56 \mathrm{~ms}, \theta_{0}=\pi / 2$ (weak Kerr) } \\
\hline 6 & .013 & -5.686 & 1.485 & 1.137 & .521 & 5.692 & 1.475 & 1.139 & .823 \\
\hline 7 & .015 & -5.367 & 1.396 & 1.113 & .578 & 5.574 & 1.385 & 1.112 & 581 \\
\hline 8 & .017 & -5.482 & 1.335 & 1.096 & .624 & 5.480 & 1.324 & 1.099 & .626 \\
\hline 9 & .019 & -5.418 & 1.291 & 1.083 & .660 & 5.426 & 1.279 & 1.086 & .664 \\
\hline 10 & .020 & -5.368 & 1.258 & 1.073 & .690 & 5.377 & 1.245 & 1.076 & .604 \\
\hline
\end{tabular}

Table 4

\begin{tabular}{|c|c|c|c|c|c|c|c|c|c|}
\hline $\bar{R}(\mathbf{k m})$ & $\overline{V_{-} / c}$ & $\varphi_{0}\left(+5^{\circ}\right)$ & $Z^{+}$ & $\Delta^{+}$ & $I_{r}^{+} / I_{\bullet}$ & $\varphi_{0}\left(-5^{\circ}\right)$ & $Z^{-}$ & $\Delta^{-}$ & $I_{1}^{-} \mid I_{0}$ \\
\hline \multicolumn{10}{|c|}{$P=1.56 \mathrm{~ms}, \theta_{0}=\pi / 2($ weak Kerr $) \quad r_{0 \mathrm{~m}}=5 R$} \\
\hline 6 & .434 & -4.770 & 3.085 & .937 & .362 & 4.948 & 2.641 & 1.008 & 380 \\
\hline 7 & .500 & -4.519 & 3.922 & .885 & 318 & 4.715 & 3.351 & 0.964 & .328 \\
\hline 8 & .567 & -4.252 & 5.239 & .838 & .270 & 4.463 & 4.486 & 0.915 & 272 \\
\hline 9 & .634 & -3.596 & 7.377 & .771 & .222 & 4.177 & 6.400 & 0.858 & .218 \\
\hline $1 \mathrm{C}$ & .701 & -3.621 & 11.073 & .703 & 177 & 3.845 & 9.767 & 0.793 & 168 \\
\hline \multicolumn{10}{|c|}{$P=1.56 \mathrm{~ms}, \theta_{1}=\pi / 2($ Schwarzschild $)$} \\
\hline 6 & & -5.375 & 1.912 & 1.075 & .453 & & 1.523 & 1.075 & .568 \\
\hline 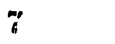 & & -5.312 & 2.120 & 1.063 & 418 & & 1.630 & 1.063 & .643 \\
\hline 8 & & -5.266 & 2.444 & 1.053 & 369 & & 1.815 & 1.053 & .497 \\
\hline 9 & & -5.231 & 2.949 & 1.046 & .310 & & 2.115 & 1.046 & .432 \\
\hline 10 & & -5.203 & 3.771 & 1.041 & .245 & & 2.611 & 1.041 & .354 \\
\hline \multicolumn{10}{|c|}{$P=1.56 \mathrm{~ms}, \theta_{8}=\pi / 2$ (weak Kerr) } \\
\hline 6 & .020 & -5.368 & 1.258 & 1.073 & .690 & 5.377 & 1.245 & 1.076 & .694 \\
\hline 7 & .024 & -5.304 & 1.218 & 1.060 & .730 & 5.315 & 1.203 & 1.064 & .735 \\
\hline 8 & .027 & -5.258 & 1.190 & 1.050 & .762 & 5.270 & 1.173 & 1.085 & .767 \\
\hline 9 & .030 & -5.221 & 1.169 & 1.043 & .785 & 5.234 & 1.151 & 1.048 & .792 \\
\hline 10 & .033 & -5.192 & 1.154 & 1.037 & .805 & 5.207 & 1.134 & 1.043 & .812 \\
\hline
\end{tabular}

to reduce the widening of the pulsar beam due to space-time curvature in longitude. We call it beam squeezing. As a result $\Delta<1$ and $\epsilon>1$. This happens once $r_{\mathrm{em}}>30 \mathrm{~km}$. Although this would imply an increase in the pulse intensity over that of emission, as was pointed out by Bhatia et al. (1988), the transverse Doppler redshift arrests this increase to keep the profile flattened for even larger $r_{\mathrm{em}}$. For slower pulsars, the squeezing effect will become pronounced at large distances from the surface where there is virtually no divergence of the beams. Thus our earlier conclusion that the brightness temperature is larger than that inferred obeervationally by about an order of magnitude (Kapoor and Datta
1985) remains tenable for millisecond pulsars whatever the location of the emission region and, for slow pulsars, it will hold if $r_{\mathrm{em}}<20 \mathrm{~km}$.

To conclude, the contribution of relativistic effects to the duty cycle and beaming can be properly gauged only when the radial location of the radio emiscion is specified. In general, the beam for all pulsars is widened in longitude as well as latitude, but the effect is significant only for $r_{\mathrm{em}}<2 R$, being stronger for the faster pulsars. For $r_{\mathrm{em}}>30 \mathrm{~km}$, it is virtually non-existent. For fast pulsars aberration will tend to reduce the duty cycle even though the beam width in latitude remains unaffected. 